\title{
LAND SURFACE PROCESSES ANALYSIS USING SENTINEL-3 OLCI AND MODIS DATA
}

\author{
Jose Gómez-Dans ${ }^{1,2}$, Gerardo López-Saldaña ${ }^{3}$, Philip Lewis ${ }^{1,2}$, Jonathan Styles ${ }^{3}$ and Pierre-Philippe Mathieu ${ }^{4}$
}

\begin{abstract}
This communication describes the optical processing chain to use Sentinel-3 OLCI and MODIS data as part of the ESA funded Synergy project of the Scientific Exploitation of Sentinel Missions (SEOM) component of the EO Envelope programme. One of the goals of the project is to use Data Assimilation techniques to produce land surface products combining the data from Sentinels-2 and 3. Some of the derived products are the OLCI atmospherically corrected data that can be used to generate a spectral BRDF product from OLCI and MODIS, broadband albedo and different vegetation parameters. The project also implements a series of efficiency improvements to the algorithms to speed up the processing. The demonstrator product uses one year of OLCI and MODIS data (2017).
\end{abstract}

\section{INTRODUCTION}

The advent of initiatives like ESA's Climate Change Change Initiative (CCI), similar moves by other space agencies, as well as the increased number of applications that require frequent, gap-free and continuous monitoring of the land surface have resulted in an interest in consistently combining observations from heterogeneous sensors in a way that makes them compatible for quantitative applications. This usually means that the observations are interpreted in terms of land surface parameters, such as albedo, leaf area index (LAI), or soil and leaf optical properties. It is of critical importance that the inferences of these parameters are internally consistent, and are quantified by an estimation of uncertainty [1], [2].

As part of this process, the effect of the atmosphere on each acquisition needs to be compensated. Clouds need to be flagged, and the surface directional reflectance over the sensor's spectral bands needs to be inferred. This process in essence converts the top of atmosphere (TOA) Level $1 / 1 \mathrm{C}$ product to a bottom of atmosphere (BOA) Level 2/2A product.

Recent approaches to atmospheric correction have started exploiting the relatively slow variation in land surface reflectance compared to the fast variation in atmospheric parameters. Methods such as those proposed by [3], [4], [5] succesfully make use of this observation. In the current work, we build on these foundations to provide a novel, uncertainty quantified, multi-sensor land surface monitoring framework. The main ingredients of this system include (i) a simple but flexible model of evolution of the land surface

*This work was supported by ESA contract 4000112388/14/I-NB and H2020 project MULTIPLY 687320

${ }^{1}$ National Centre for Earth Observation, NERC, UK

${ }^{2}$ Dept of Geography, University College London, UK

${ }^{3}$ Assimila Ltd, Reading, UK

${ }^{4}$ European Space Agency, Frascati, Italy properties; (ii) use of widely available predictions of atmospheric composition provided by meteorological centers such as Copernicus Atmospheric Monitoring Service (CAMS); (iii) spectral transformations between different sensors to allow data blending; and (iv) the use of advanced physical models of the atmosphere to infer both atmospheric composition and land surface BRDF in a set of common spectral narrow bands. These so-called spectral BRDF predictors can then be used further to calculate broad band bi-hemispheric reflectance (BHR), and then further used in an inversion scheme to retrieve land surface parameters such as effective LAI and leaf and soil optical properties.

In this communication, the system outlined above is presented, and demonstrated with a combination of S3/OLCI and MODIS data. The atmospheric correction of the individual sensors is introduced in $\S$ III. The spectral transformations are shown in $\S \mathrm{IV}$. The non-linear inversion of land surface parameter is shown in $\S \mathrm{V}$

\section{DATA PREPROCESSING}

$\begin{array}{lll}\text { OLCI } & \text { radiances were acquired from } \\ \text { the Sentinel-3 Pre-Operations Data Hub }\end{array}$ (https://scihub.copernicus.eu/s3). The full resolution top of atmosphere product is composed of 22 measurement data files: 21 files containing radiances for each band at approximately $300 \mathrm{~m}$ (one band per file), accompanied by the associated error estimates, plus an additional file providing all data related to pixels that have been removed during the re-sampling process. Additionally, it contains the geometry data (solar and azimuth angles). L1 OLCI data for late 2016 and the whole 2017 covering two different MODIS tiles, h18v04 covering the Alps and tile h17v05 that covers Southern Spain and Northwest Africa were downloaded. TOA radiances were transformed into TOA reflectances using the S3 Toolbox, then all granules were mosaicked to get a single image per day and reprojected to $500 \mathrm{~m}$ and resampled to match the MODIS Sinusoidal Grid.

\section{ATMOSPHERIC CORRECTION}

A number of atmospheric correction schemes are based on the assumption of a Lambertian land surface BRDF. This assumption leads to significant errors in the corrected surface directional reflectance in situations with very oblique geometries, or when aerosol loading is high [6]. In this case, we use linear BRDF kernel models [7], [8] to describe the land surface anisotropy and we couple the atmosphere and land surface explicitly. As such, the TOA radiance at a given 

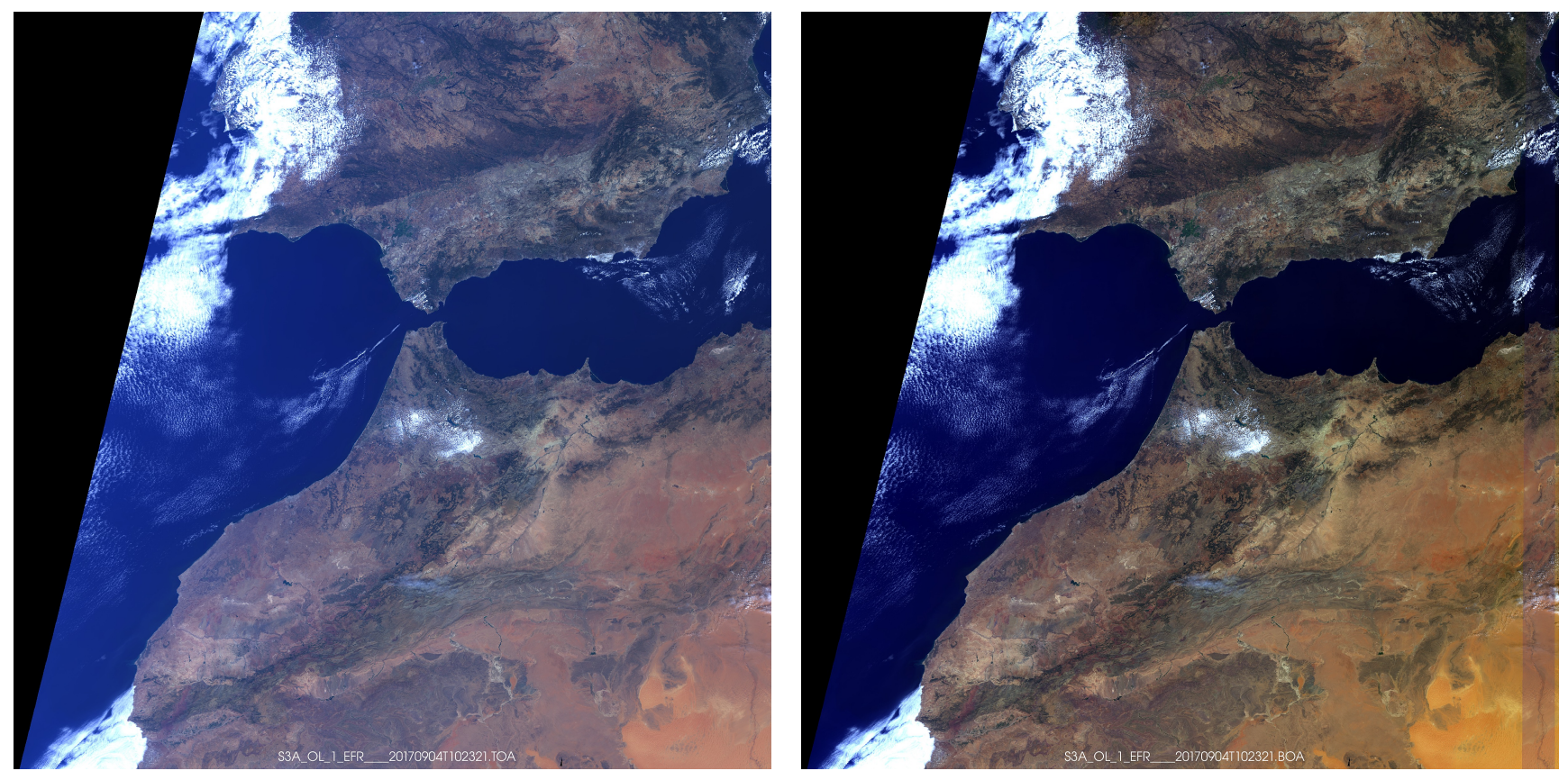

Fig. 1: OLCI TOA (left) and BOA (right) reflectance for tile h17v05

wavelength and view/illumination geometries is given by the 3 linear kernel parameters or spectral BRDF descriptors, the atmospheric composition (e.g. aerosol optical thickness, total columnar water vapour, $\mathrm{O}_{3}$ concentration). The $6 \mathrm{SV} 1$ atmospheric radiative transfer model [9] is used to produce this mapping. The system assumes that the mapping between TOA and BOA reflectances is only hampered by additive Gaussian noise with a known variance (and no bias), which results in a normal likelihood function, $p(\vec{y} \mid \vec{x})$,

$$
\log (p(\vec{y} \mid \vec{x})) \propto \frac{1}{2}[H(\vec{x})-\vec{y}]^{\top} \mathbf{C}_{o b s}^{-1}[H(\vec{x})-\vec{y}] .
$$

In this equation, $H$ is the radiative transfer model, $\mathbf{C}_{o b s}$ is the observational error covariance matrix, $\vec{y}$ are the observations (TOA reflectance or radiance), and $\vec{x}$ is the state, a combination of the three linear BRDF kernel weights for each spectral band, and the atmospheric composition parameters. Maximising the log-likelihood in Eq. 1 as a function of $\vec{x}$ results in an ill-posed problem [10], [11], which in this case is also constrained with information on atmospheric composition from the Copernicus Atmospheric Monitoring Service (CAMS, http://atmosphere.copernicus.eu). An additional source of prior information is the use of the estimate of the kernel parameters from the previous time-step (currently, we use a daily timestep), with a small inflation on the uncertainty, in effect assuming that the land surface paramters only change slowly in time [12].

Minimising the compound cost function results in an nonlinear least squares method, which in this case is solved via local linearisations of $H(\vec{x})$ (which in this case is the 6S RT model) using the gradient of the RT model. For the sake of efficiency, this is done using Gaussian process (GP) emulators [13]. The solution of this step results in an inference on the per-band BRDF kernel weights, as well as the atmospheric composition parameters. Fig 1 depicts a True Colour (bands 9,6,3 RGB) example of the OLCI atmospheric correction for September 4, 2017.

\section{SPECTRAL TRANSFORMATIONS}

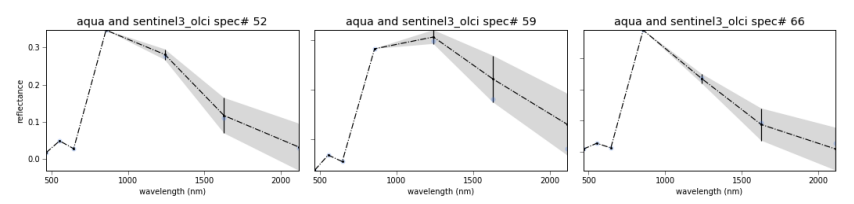

Fig. 2: Examples of spectral transformations: MODIS reflectance predicted from OLCI bands.

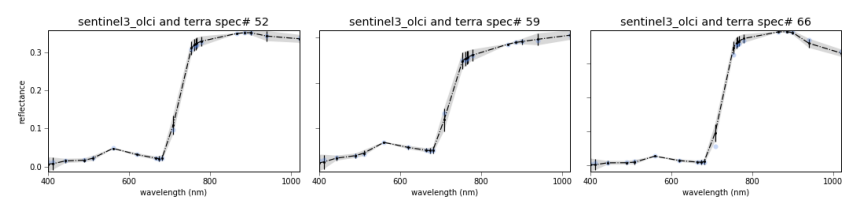

Fig. 3: Examples of spectral transformations: OLCI reflectance predicted from MODIS bands.

The approach outlined in the previous Section is not particularly new and shares a lot with the approach proposed by [14], only here we take a more formal Bayesian view of prior distributions. To extend this approach to combinations of different sensors, we suggest that defining a set of linear kernel weights in an spectrally informative set of narrow bands, and then provding a set of transformations from these bands to individual bands of actual sensors (and viceversa) allows for a simple blending framework. In 


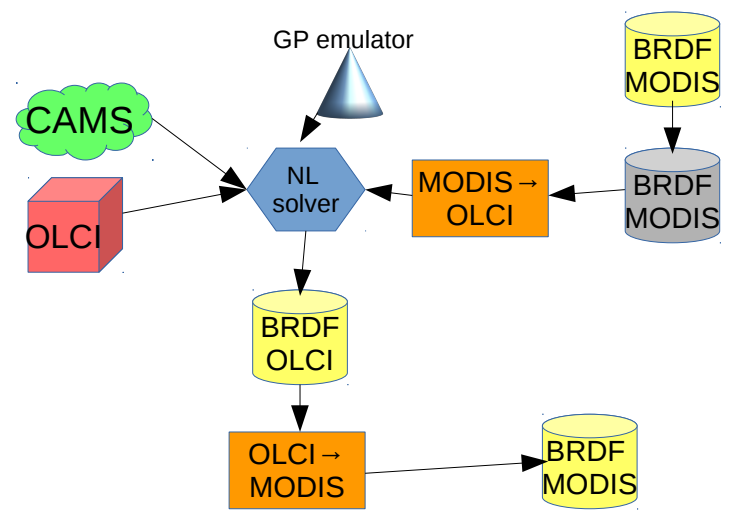

Fig. 4: Schematic of atmospheric processing chain. Spectral BRDF descriptors in the MODIS bands are used to provide a prior distribution for kernel weights in the target (in this case, OLCI) sensor bands using linear transformations. Prior atmospheric parameter distributions are obtained from CAMS, and emulators of the $6 \mathrm{~S}$ model are used to invert both atmospheric parameters and BRDF kernel weights in OLCI spectral space. These are then transformed back to MODIS space, and used for subsequent processing.

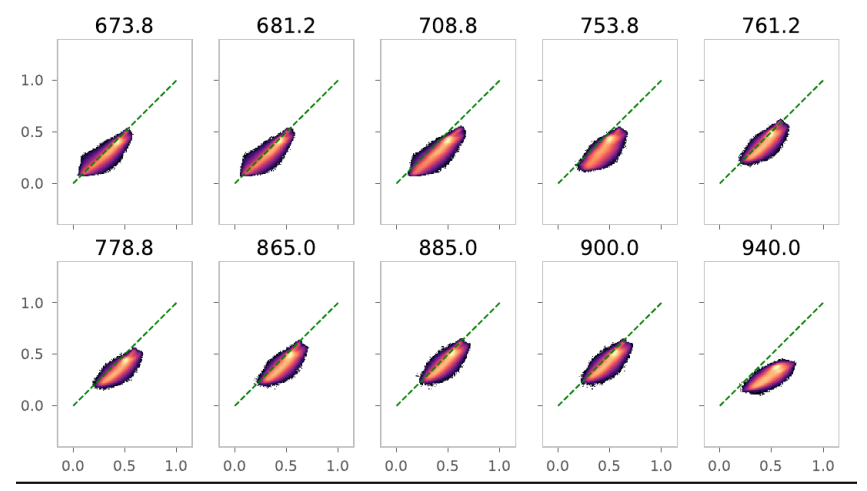

Fig. 5: Comparison of OLCI TOA reflectance with predictions from MODIS using the spectral transforms

practice, BRDF descriptors are stored in the 7 MODIS 500m bands. These provide a compact yet informative description of most spectral features. When observations from a given sensor need to be ingested, a prior land surface distribution of the sensor parmaters is used using a transformation of the MODIS BRDF descriptors to the spectral bands of the new sensor. The prior distribution derived from e.g. CAMS is also produced, and both atmospheric parameters and linear kernel weights in the native sensor spectral bands are inferred (together with relevant uncertainties). The native linear kernel weights (and associated uncertainties) are then converted to the target spectral set, and used for further processing. The scheme is showed schematically in Fig. 4

Using a spectral database and using the spectral response functions for the different sensors, we produce a set of linear mappings that allow one to convert observations acquired by the MODIS (... OLCI) sensor to those acquired by OLCI (... MODIS) sensor. The linear mappings are stored in a matrix $\mathbf{T}$, and in order to predict OLCI reflectances in one band $\lambda_{O}$ and geometry, we can use the linear kernel weights in the different MODIS bands and transform them into the target MODIS reflectance as:

$\rho_{O}\left(\Omega_{O}, \Omega_{O}^{\prime}, \lambda_{O}\right)=\mathbf{T}_{M \rightarrow S 3} \cdot\left[\begin{array}{c}\sum_{i} f_{i}\left(\lambda_{M, 1}\right) \cdot K_{i}\left(\Omega_{O}, \Omega_{O}^{\prime}\right) \\ \vdots \\ \sum_{i} f_{i}\left(\lambda_{M, 7}\right) \cdot K_{i}\left(\Omega_{O}, \Omega_{O}^{\prime}\right)\end{array}\right]$

Some examples of these transformations can be seen in Figs. 2 3, showing that with the linear transformations appear to provide a reasonable, uncertainty quantified predictions of surface reflectance. Note how the e.g. the prediction of the $940 \mathrm{~nm}$ band in OLCI from MODIS has a large uncertainty (due to strong atmospheric absorption effects), and that the prediction of the MODIS SWIR bands is also characterised by a large uncertainty, due to OLCI not sampling that spectral area.

In Fig 5, we show comparisons of the OLCI TOA reflectance againt a prediction of the BOA reflectance coming from the MODIS processing chain. This shows a mostly linear behaviour, except in the strong water vapour absorption band.

\section{APPLICATIONS}

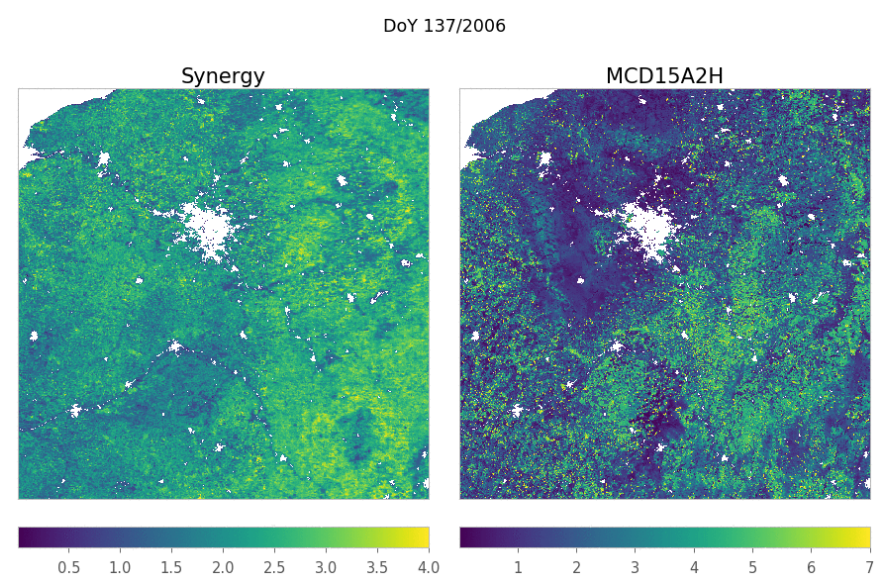

Fig. 6: Examples of retrieved effective leaf area index (left) using the proposed approach on MODIS data and the official MODIS LAI product (right)

The generated spectral BRDF descriptors can be used for several applications. They can be integrated spectrally and angularly to estimate e.g. broadband bi-hemispherical reflectance (BB BHR, or white sky albedo). BB BHR can be interpreted using a suitable RT model, such as 2Stream. Under the assumption of Gaussian uncertainty in BB BHR (remember that uncertainty is propagated from the Level 1C product to the kernel weights and thus to BB BHR), we end up with a similar cost function to that shown in Eq. 1. where $H(\vec{x})$ stands for the 2Stream RT model, and the vector 
of parameters includes effective LAI, leaf reflectance and transmittance in the visible and near infrared broadbands, as well as soil albedo in the visible and near infrared broadbands. We assume the same priors as Pinty [15] for the parameters. Again, the cost function is minimised though a linearisation of the RT model using emulators [13]. This is numerically much more efficient than solving the nonlinear problem, even though the linearisation requires a few iterations (usually no more than five or six) to converge to the solution. Another potential application that will be tested is the use of the high temporal resolution of the coarse resolution synergy products (S3/OLCI and MODIS), that using the same approach described in III can constrain the atmospheric correction of S2 data.

\section{CONCLUSIONS}

In this contribution, we present a method to combine observations from different sensors in a consistent way, to produce a continuous set of land surface parameters (with associated uncertainties). The method also produces state of the art atmospherically corrected surface directional reflectance and cloud clearing. The system makes use of additional global products (e.g. CAMS atmospheric composition) to simplify the rather complicated inverse problem that results from the fully-coupled land-atmosphere atmospheric correction scheme. The Bayesian nature of this approach leads to uncertainty quantified estimates of spectral BRDF descriptors, which can then be used to calculate important magnitudes such as white sky albedo and, by inversion of an RT model, biophysical land parameters.

It is important that many of the steps in this approach are linear and are solved using standard linear tools. This is important as uncertainty propagation is very simple. Nonlinear parts are solved using linearisations that exploit the fast emulators [13] for the costly RT models and their ability to quickly and accurately predict the Jacobian of the model. These techniques allow the approach to scale to large numbers of pixels, as well as exploiting parallel architectures, withot resorting to re-writing RT codes (and hence adding flexibility to the choice of RT model used).

\section{REFERENCES}

[1] Christopher J Merchant, Frank Paul, Thomas Popp, Michael Ablain, Sophie Bontemps, Pierre Defourny, Rainer Hollmann, Thomas Lavergne, Alexandra Laeng, Gerrit de Leeuw, Jonathan Mittaz, Caroline Poulsen, Adam C Povey, Max Reuter, Shubha Sathyendranath, Stein Sandven, Viktoria F Sofieva, and Wolfgang Wagner, "Uncertainty information in climate data records from Earth observation," Earth System Science Data, vol. 9, no. 2, pp. 511-527, July 2017.

[2] A C Povey and R G Grainger, "Known and unknown unknowns: uncertainty estimation in satellite remote sensing," Atmospheric Measurement Techniques, vol. 8, no. 11, pp. 4699-4718, Nov. 2015.

[3] Shunlin Liang, Bo Zhong, and Hongliang Fang, "Improved estimation of aerosol optical depth from MODIS imagery over land surfaces," Remote Sensing of Environment, vol. 104, no. 4, pp. 416-425, 10 2006.

[4] A Lyapustin, Y Wang, I Laszlo, R Kahn, S Korkin, L Remer, R Levy, and JS Reid, "Multiangle implementation of atmospheric correction (maiac): 2. aerosol algorithm," Journal of Geophysical Research: Atmospheres, vol. 116, no. D3, 2011.
[5] Olivier Hagolle, Mireille Huc, David Pascual, and Gerard Dedieu, "A multi-temporal and multi-spectral method to estimate aerosol optical thickness over land, for the atmospheric correction of FormoSat-2, LandSat, VENS and sentinel-2 images," Remote Sensing, vol. 7, no. 3, pp. 2668-2691, 32015.

[6] Miguel O Román, Crystal B Schaaf, Philip Lewis, Feng Gao, Gail P Anderson, Jeffrey L Privette, Alan H Strahler, Curtis E Woodcock, and Michael Barnsley, "Assessing the coupling between surface albedo derived from MODIS and the fraction of diffuse skylight over spatially-characterized landscapes," Remote sensing of environment, vol. 114, no. 4, pp. 738-760, Apr. 2010.

[7] W Lucht and J L Roujean, "Considerations in the parametric modeling of BRDF and albedo from multiangular satellite sensor observations," Remote Sensing Reviews, vol. 18, no. 2-4, pp. 343-379, 2000.

[8] W Lucht and P Lewis, "Theoretical noise sensitivity of BRDF and albedo retrieval from the EOS-MODIS and MISR sensors with respect to angular sampling," International journal of remote sensing, vol. 21, no. 1, pp. 81-98, 2000.

[9] E F Vermote, D Tanre, J-L Deuze, M Herman, and J-J Morcette, "Second Simulation of the Satellite Signal in the Solar Spectrum, 6S: an overview," IEEE transactions on geoscience and remote sensing: a publication of the IEEE Geoscience and Remote Sensing Society, vol. 35, no. 3, pp. 675-686, May 1997.

[10] D S Kimes, Y Knyazikhin, J L Privette, A A Abuelgasim, and F Gao, "Inversion methods for physicallybased models," Remote Sensing Reviews, vol. 18, no. 2-4, pp. 381-439, 2000.

[11] B Combal, F Baret, M Weiss, A Trubuil, D Macé, A Pragnère, R Myneni, Y Knyazikhin, and L Wang, "Retrieval of canopy biophysical variables from bidirectional reflectance: Using prior information to solve the ill-posed inverse problem," Remote sensing of environment, vol. 84, no. 1, pp. 1-15, 2003.

[12] T Quaife and P Lewis, "Temporal constraints on linear BRDF model parameters," IEEE transactions on geoscience and remote sensing: a publication of the IEEE Geoscience and Remote Sensing Society, vol. 48, no. 5, pp. 2445-2450, 2010.

[13] José Gómez-Dans, Philip Lewis, and Mathias Disney, "Efficient Emulation of Radiative Transfer Codes Using Gaussian Processes and Application to Land Surface Parameter Inferences," Remote Sensing, vol. 8, no. 2, pp. 119, Feb. 2016.

[14] Alexei Lyapustin, Yujie Wang, Istvan Laszlo, Thomas Hilker, Forrest G Hall, Piers J Sellers, Compton J Tucker, and Sergey V Korkin, "Multiangle implementation of atmospheric correction for modis (maiac): 3 . atmospheric correction," Remote Sensing of Environment, vol. 127, pp. 385-393, 2012.

[15] B Pinty, I Andredakis, M Clerici, T Kaminski, M Taberner, M M Verstraete, N Gobron, S Plummer, and J-L Widlowski, "Exploiting the MODIS albedos with the Two-stream Inversion Package (JRC-TIP): 1. Effective leaf area index, vegetation, and soil properties," Journal of geophysical research, vol. 116, no. D9, pp. D09105, May 2011. 